\title{
Research on cloud computing high-density data center infrastructure and environment matching technology
}

\author{
Lianteng Shen ${ }^{1, *}$, Shengpan Qian ${ }^{1}$, Tianyi Zhai ${ }^{1}$,Ling $\mathrm{Li}^{1}$, and Zhe $\mathrm{Li}^{1}$ \\ ${ }^{1}$ China Electric Power Research Institute, 100192, Beijing, China
}

\begin{abstract}
The current rapid development of cloud computing and networks has put forward new requirements for the construction of new infrastructure such as data centers. This paper compares traditional data centers and high-density data centers, proposes a three-tier infrastructure for high-density data centers, and analyzes the data center environment. To solve the obvious problem of software and hardware heterogeneity in high-density data centers, this paper uses virtualization technology to pool resources in high-density data centers and introduces SOA architecture to manage software and hardware resources hierarchically. Finally, the data center infrastructure and environment matching technology are studied.
\end{abstract}

\section{Introduction}

Under the wave of the new generation of the digital economy, various new business models have achieved great development, and new technologies such as 5G, Internet of Things, cloud computing, big data, artificial intelligence, etc., which carry these new services, have led to a solid foundation for the Internet Of ultra-large-scale data centers have entered explosive development. On March 4, 2020, the Standing Committee of the Political Bureau of the Central Committee pointed out that to speed up the construction of new infrastructure such as $5 \mathrm{G}$ and data centers, the importance of "new infrastructure" is self-evident, bringing new opportunities and opportunities for the construction of big data centers. challenge. The construction of data centers is an important part of the "new infrastructure" and the key to the transformation and upgrading of the power grid to the energy Internet. With the continuous construction of the State Grid's large cloud and mobile, to avoid the rapid expansion of data centers due to the explosive increase in infrastructure brought about by application growth, and the consequent sharp increase in various operation and maintenance costs, smart grid applications are showing high performance-intensive development trend. Data centers are required to develop in the direction of small-scale, high-density computing and storage networks, and more optimized performance. Therefore, high-density data centers have become the mainstream construction trend of future data centers.

\footnotetext{
* Corresponding author: zhaitianyi@epri.sgcc.com.cn
} 
The current research on high-density data center infrastructure mainly focuses on the overall data center architecture and virtualization technology research. Literature [1] analyzes the power data center infrastructure and key technologies based on cloud computing and deeply analyzes the logic of the power data center Architecture and application situation. Literature [2] Proposed a cloud computing-based power data center infrastructure, based on Hadoop, designed a power data center cloud computing platform. Literature [3] took university data centers as an example and proposed software-based on hyper-converged architecture define the data center design plan. Literature [4] proposed a cloud computing data center infrastructure based on virtualization and distributed technology, studied its key technologies, and designed a construction plan that conforms to the status quo of power companies. Literature [5] studied data centers key energy-saving technologies, in-depth discussion of key energy-saving technologies such as data center power system optimization, and refrigeration system optimization. Literature [6] introduced the application of virtualization technology in the power industry, designed server, storage, and application pooling solutions, and the results are evaluated. Literature [7] studied the optimization strategy of power supply and heat dissipation in modern high-density data center universities. Literature [8] designed a high-density data center refrigeration and air-conditioning system based on a specific data center, and provided new ideas. Literature [9] analyzed the shortcomings of traditional data centers and designed a new network architecture with snowflake structure. In summary, the current research on high-density data centers is mainly focused on key technologies such as infrastructure deployment and resource virtualization, and how the infrastructure of high-density data centers is related to the electrical resource environment and HVAC environment. Research on matching and improving the use of data centers under conditions of narrow space has become blank. This article analyzes the infrastructure and environment of high-density data centers, and compares them with traditional data centers, and analyzes the advantages and disadvantages of high-density data centers. Utilization virtualization technology pools data center resources, and proposes integrated management of data center software and hardware resources based on the SOA model to solve the problems of obvious heterogeneity of high-density data center hardware and low resource utilization. Finally, the article mainly compares and analyzes The key technology for matching and optimizing the high-density data center infrastructure architecture with the data center's HVAC environment and electric resource environment is used to improve the deployment capabilities of high-density data centers and reduce energy consumption.

\section{High-density data center infrastructure and environment analysis}

A high-density data center usually refers to a data center with a single cabinet power load above $8 \mathrm{KW}$ and densely placed, which can integrate more servers in a smaller space, provide stronger I/O expansion capabilities, and adopt ultra-high The hybrid and cooperative infrastructure of performance hardware (such as NVRAM, GPU) and general-purpose hardware realizes the integration of higher computing, storage capabilities, and infrastructure density. Because of its small space, dense placement, and high power, higher requirements are placed on the physical space to ensure its reliability and availability. 


\subsection{Analysis of traditional data center environment}

The high-density data center carries many servers, but it is not just a collection of servers. Its system is complex, including various data communication equipment, environmental control equipment, monitoring equipment, and other security equipment and other important equipment, which has very strict requirements on the data center environment.

Overall, the traditional data center environment is divided into five dimensions, namely, water resources environment, electricity resources environment, security environment, network environment, and geographic environment[10]. This article focuses on analyzing the water resources environment and electricity resources environment of the data center. Water resources environment refers to the water source for cooling the data center. Commonly used water sources include circulating water for cooling the air conditioning system of the computer room when the outdoor wet-bulb temperature is low enough or outdoor low-temperature natural water sources. The data center generates a lot of heat when using natural water sources for cooling, which will have a certain impact on the water temperature of nearby waters, and even cause pollution. Therefore, when using natural water sources for cooling, the impact of warm drainage on the water resources of the data center should be fully considered. Ensure the availability and safety of the natural water cooling system in the data center. The electrical resource environment refers to the electrical resources of the data center to provide power for the data center. As energy conservation becomes a key factor in the construction of the data center, the electrical resource environment has become one of the important environments for the data center.

\subsection{Analysis of high-density data center infrastructure}

The high-density data center infrastructure is divided into three layers, namely, the infrastructure architecture layer, the IT hardware architecture layer, and the cloud platform architecture layer. Compared with traditional data centers, the IT hardware architecture layer of high-density data centers is quite different. The infrastructure of the high-density data center still uses the traditional wind, fire, and hydropower system, but it puts forward new requirements such as stronger heat dissipation capacity, higher power supply reliability, and lower energy consumption. The core of them is heating, ventilation, and cooling. The system, electrical system, and monitoring system. The IT hardware architecture layer mainly includes hardware devices including servers, storage devices, networks, and other hardware, which are the basic resources for cloud computing. High-density data centers use ultra-high-performance hardware such as NVRAM and GPU. The heterogeneity is obvious. In order to achieve higher performance in a small space, modular structures are often used, which is quite different from traditional data centers. The IT hardware architecture level needs to focus on realizing the control capabilities of software-defined technology on the IT layer to meet the needs of multi-source heterogeneous applications for servers, storage devices, and networks. The main technologies of the cloud platform architecture layer are virtualization pooling technology and resource management technology. Pooling technology mainly involves computing resource pools, storage resource pools, and network resource pools. Another key technology of the high-density data center cloud platform architecture is the resource management system, which mainly realizes unified management of physical equipment, virtualized resource pool management, automated deployment, high availability of resource pools, business-based intelligent management, and control capabilities and interfaces. The data center infrastructure is shown in Figure 1. 

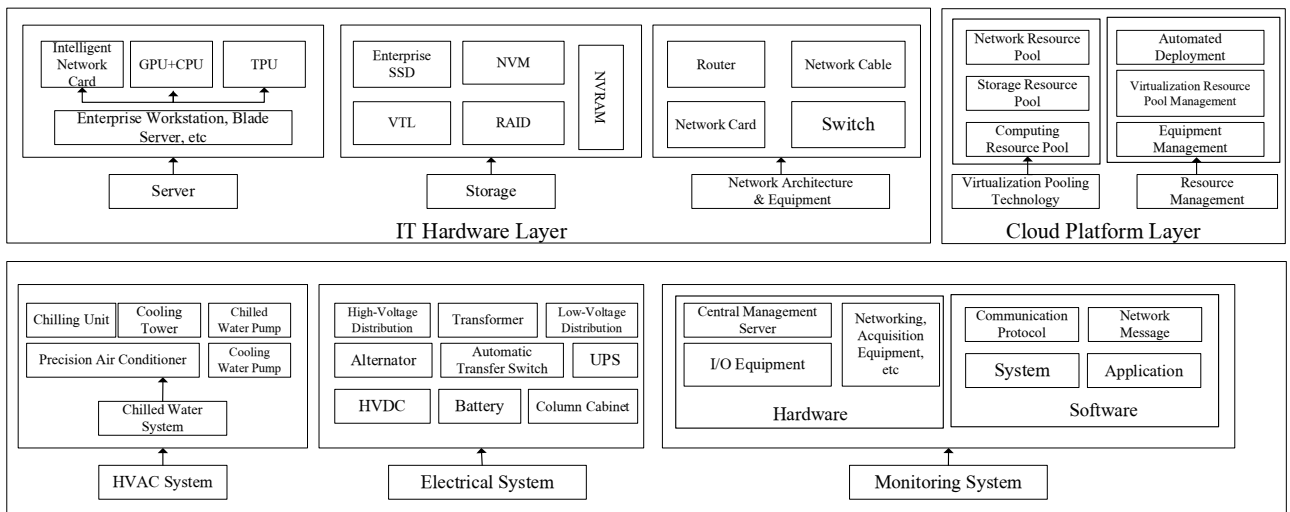

Infrastructure Layer

Fig. 1. High-density data center infrastructure diagram.

\subsection{Comparative analysis of high-density data centers and traditional data centers}

Compared with the traditional data center, the high-density data center adopts an infrastructure based on the hybrid coordination of ultra-high-performance hardware (such as NVRAM, GPU) and general-purpose hardware to achieve higher integration of computing, storage capabilities, and infrastructure density. Compared with traditional data centers, high-density data centers have obvious advantages in terms of performance and space. Traditional data center infrastructure adopts independent construction methods of general hardware (such as SAS, SATA, x86, etc.), which sacrifices overall performance in order to achieve universality. Its responsiveness has become the bottleneck of business system transformation and rapid development, and ultra-high-performance hardware is introduced After building a high-density architecture, its infrastructure design presents a hybrid heterogeneous hardware model, and the overall performance and density of computing and storage have been improved.

Table 1. Comparison of high-density data centers and traditional data centers.

INFRASTRUCTURAL EQUIPMENT

HARDWARE

RESPONSE ABILITY

COMPUTING ABILITY

STORAGE ABLILITY

ADAPTION
TRADITIONAL DATACENTER

\begin{tabular}{|c|c|}
\hline $\begin{array}{c}\text { Wind power, hydropower, } \\
\text { thermal power generation } \\
\text { system }\end{array}$ & $\begin{array}{c}\text { Updated wind power, } \\
\text { hydropower, thermal power } \\
\text { generation system }\end{array}$ \\
\hline $\begin{array}{c}\text { General hardware (SAS, } \\
\text { SATA,X86, etc.) }\end{array}$ & $\begin{array}{c}\text { Ultra-high performance hardware } \\
\text { (NVRAM,GPU, etc.) }\end{array}$ \\
\hline Weak & Strong \\
\hline Normal & Strong \\
\hline Normal & Strong \\
\hline Strong & $\begin{array}{c}\text { Low hardware and software } \\
\text { coordination ability, low } \\
\text { universality }\end{array}$ \\
\hline
\end{tabular}




\section{Research on integrated management technology of software and hardware resources in high-density data centers}

\subsection{High-density data center resource pooling}

Virtualization technology is a technology that allows multiple operating systems to run on one physical server without interfering with each other [11]. It abstracts the physical resource architecture of the data center, builds a virtual layer, and weakens the coupling between upper and lower systems. It not only makes full use of various hardware resources but also avoids mutual interference between the upper and lower layers, which is the basis for realizing cloud computing.

The resource pooling process of a high-density data center is shown in Figure 2. First, the logic pooling technology is used to realize the resource pooling of power supply equipment and cooling equipment to form a power supply resource pool and a cooling resource pool. Secondly, the CPU, Resources such as memory, external memory, and network are virtualized to form a computing resource pool, memory resource pool, external memory resource pool, and network resource pool, decoupling different types of resources [12]. At the same time of virtualization, the relationship mapping table of resources between different resource pools is established to determine whether the resources in different resource pools belong to the same physical machine, which provides convenience and support for subsequent virtual machine assembly [13].

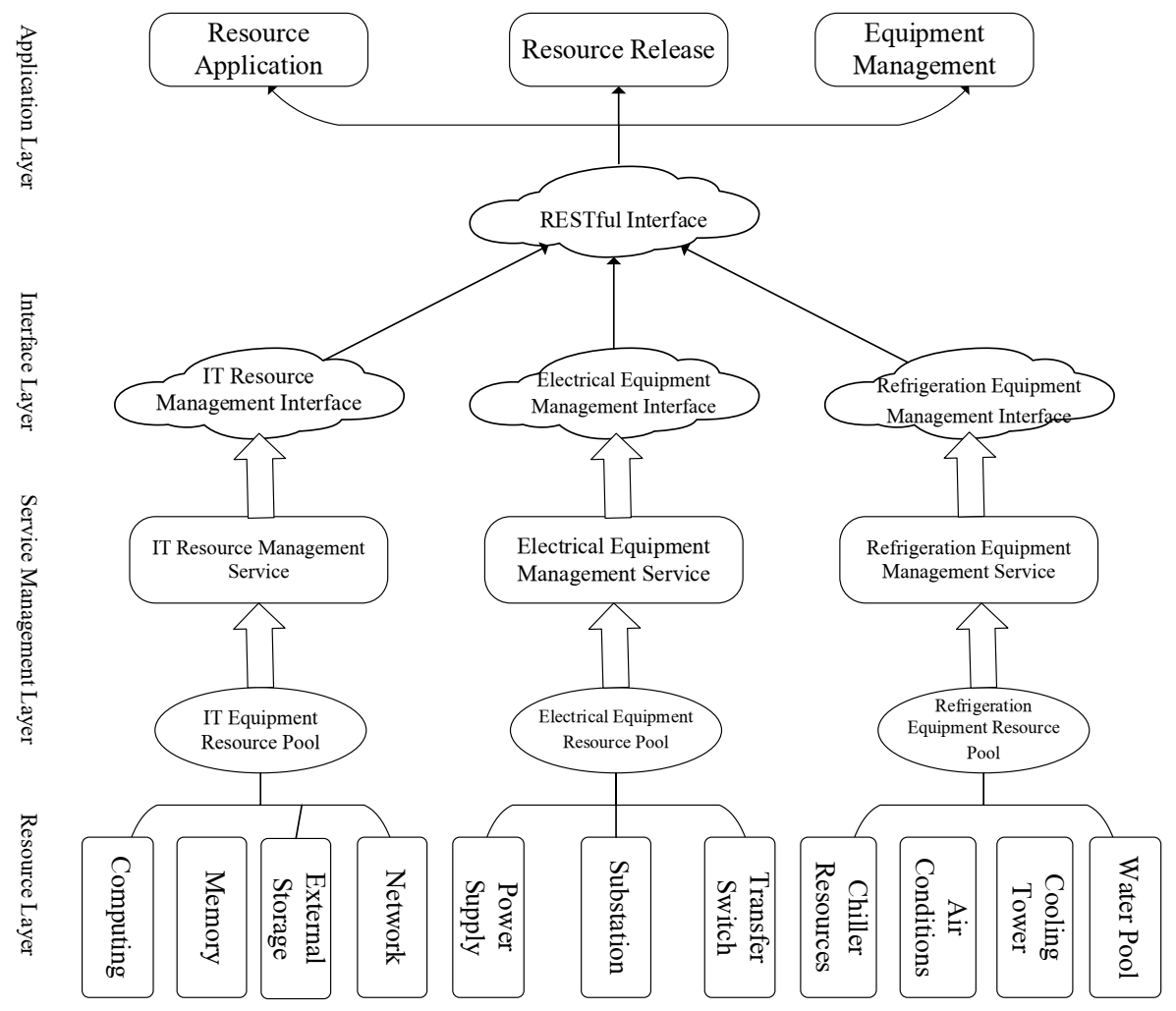

Fig. 2. Datacenter resource pooling process diagram.

Based on virtualization and decoupling, according to different data center services, resources in different resource pools are combined into virtual machines on-demand to 
meet the computing and storage resources required by the task. The physical structure of the servers in the data center limits Combinations of resources in different resource pools, in the process of assembling virtual machines, first establish a mapping relationship table and then select different resources on the same server according to the mapping relationship table to form a virtual machine that meets the task computing storage requirements. Try to avoid factors The additional computing and transmission overhead of virtual machines across physical servers.

\subsection{Software and hardware integrated management based on SOA architecture}

Virtualization-based resource pooling technology unifies all kinds of resources in high-density data centers. However, how to connect different types of resources to realize software and hardware collaborative management is still a problem that needs to be solved. The SOA architecture can uniformly deploy, manage, and use the components in the network according to requirements. On the basis of resource pooling, it can perform unified integrated management of different types and different levels of pooled software and hardware resources, with coarse-grained service interface classification, Loose coupling, and other advantages, can realize the service of pooled resource management function, provide interfaces for top-level business and lower-level resources, and provide technical support such as data center energy efficiency optimization, task scheduling, and data center infrastructure and environment matching.

The SOA management model unifies the management of the lower-level IT equipment resource pool and the infrastructure resource pool, and provides services for the upper-level commands through interfaces. At the same time, the upper-level business issues command through the interfaces to schedule the lower-level resources. Therefore, the data center resources based on the SOA model The management model can be divided into four layers, namely the data center resource layer, management service layer, interface layer, and application layer. The resource layer includes the virtualized computing resource pool, memory resource pool, external storage resource pool, network resource pool, and other hardware facility resource pools, electrical equipment resource pool, and cooling equipment resource pool after data center virtualization. The management service layer realizes the unification of the resource layer Management, to realize the allocation and recovery of resources, to encapsulate resources into services, to realize unified linkage management of IT equipment resources and electrical and refrigeration equipment, to provide services such as message queues and load distribution, to achieve load balancing, and to ensure accurate and efficient execution of tasks, reduce power consumption. The interface layer is a link between the previous and the next, providing interfaces for the application layer to realize resource requests, resource management, and other functions; at the same time, it provides the corresponding interface for the lower-level resource management and releases resource request information. The interface layer is the key to realize the linkage between business and resources. The application layer conducts unified management of the business, realizes the on-demand and reasonable allocation of resources, and makes full use of the various resources of the lower layer. 


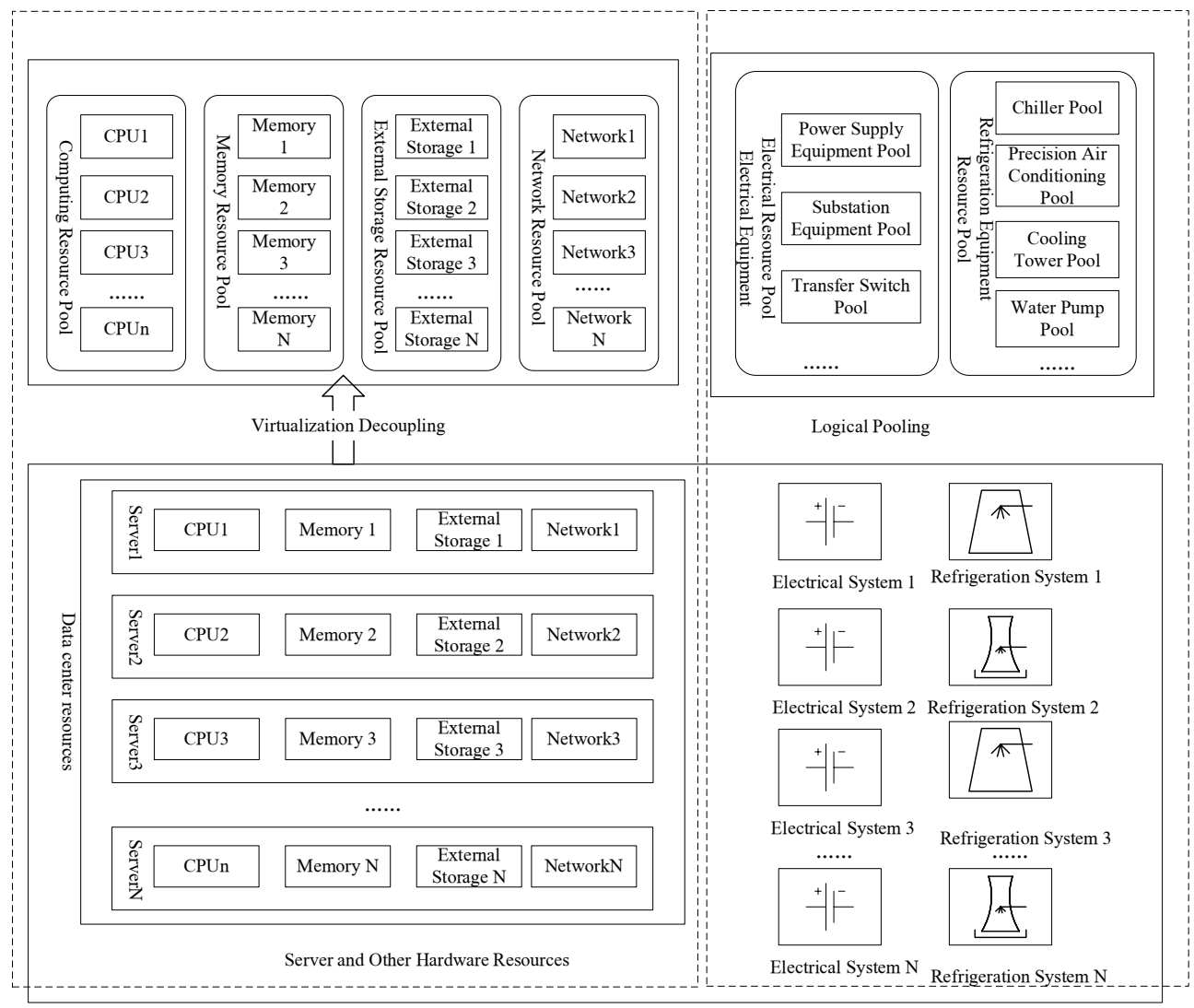

Fig. 3. Datacenter resource integrated management diagram based on SOA model.

The high-density data center uses a large number of ultra-high-performance hardware, and the heterogeneity is obvious. Through the virtualization-based resource pooling and the integrated management of data center resources, the heterogeneity of high-density data center resources is eliminated, and data center resources can be improved Utilization is of great significance to high-density data centers with small space.

\section{High-density data center infrastructure and environment matching technology research}

High-density data centers have high power loads, densely placed cabinets, and small space for use. High performance and high density also place higher requirements on the environmental protection of data centers. From the perspective of high-density data center infrastructure, this paper compares and analyzes existing matching technologies, and studies high-density data center infrastructure and environment matching technologies.

With the development of new technology and information and communication, people are pursuing more intelligent lifestyles, and the requirements for infrastructure such as data centers are also increasing. Its stable, efficient, and uninterrupted operation requires high-voltage DC technology to optimize the power system of data centers. The essential. High-density data centers mainly use high-voltage direct current technology, liquid cooling technology, and indirect evaporative cooling technology to match high-density infrastructure and environment. 


\subsection{Matching the infrastructure layer with the electrical environment of the data center}

Current data centers mostly use UPS. High-density data centers use more ultra-high-performance hardware and have a small space. Traditional UPSs cannot satisfy high-density data centers in terms of reliability, power supply efficiency, maintainability, and large area Requirements. The high-voltage direct current system (HVDC) includes an AC power distribution unit, rectifier module, battery, DC power distribution unit, battery management unit, insulation monitoring unit, and monitoring modules [14]. Its working principle is that when the mains power is normal, the rectifier module of the high voltage DC system converts the $380 \mathrm{~V}$ AC output by the AC power distribution unit into $240 \mathrm{~V}$ high voltage DC, and the high voltage DC powers the equipment through the DC power distribution unit and also charges the battery. When the mains is abnormal, the battery will supply power to the communication equipment.

The high-voltage DC system adopts a modular design, with no need to configure an independent power supply room, saving space. Parallel technology is simple, reliable, and capable of sleeping. The number of working modules can be adjusted in real-time according to business conditions. The flexibility is high. Table 2 shows the comparison of various indicators with traditional UPS.

Table 2. Performance comparison between HVDC system and traditional UPS.

PARAMETER

SYSTEM POWER

STABILITY

RELIABILITY

SECURITY

UNIVERSALITY

COST
HVDC SYSTEM

\begin{tabular}{|c|c|}
\hline High & High \\
\hline High & Low \\
\hline High & Low \\
\hline High & Low \\
\hline High & High \\
\hline Low & High \\
\hline
\end{tabular}

\subsection{The matching of high-density data center infrastructure and HVAC environment}

The high-density data center has a large single cabinet power density, which exceeds the conventional power density requirements, and puts forward higher requirements for the design of the refrigeration system. Liquid cooling technology uses liquid as a transmission medium to take away the heat of the server, reducing the air volume required by the server, and reducing the overall air supply-demand of the data center, reducing the local hot spots caused by the backflow of the computer room [15]. Liquid cooling technology effectively suppresses the instantaneous increase in the internal temperature of CPU and other components, to a certain extent, allows to increase the density of server deployment, improve the integration of data centers, and become the best match for the HVAC direction of high-density data centers.

At present, there are three main types of liquid-cooled refrigeration in data centers, cold plate, immersion, and spray [15]. The cold plate type is relatively mature at present, and the immersion type is divided into two types, single-phase liquid cooling and phase change liquid cooling, and is currently in its infancy. The spray type is currently low in popularity. 
Existing data centers mostly use traditional HVAC systems. This article compares the existing data centers of State Grid with the high-density data centers using liquid cooling technology in terms of liquid-cooled server maintenance methods, refrigerants, civil engineering facilities, and costs., as shown in Table 3.

The liquid cooling technology uses a large specific heat capacity and higher heat dissipation efficiency, which can reduce energy consumption and save costs, which is of great significance to the improvement of PUE. Compared with traditional air cooling, the same volume of liquid takes away heat 3000 times that of the same volume of air, and the liquid's thermal conductivity is 25 times that of air. The noise reduction effect is better. At the same heat dissipation level, the noise of the liquid cooling system is lower than that of the air cooling. 10-15dBA; more energy-efficient, the liquid cooling system saves about $30 \%$ of electricity than the air-cooled system[16], becoming an important means of matching high-density data center infrastructure and data center HVAC environment.

Table 3. Comparison of liquid cooling modes

\begin{tabular}{|c|c|c|c|}
\hline SUBJECT & $\begin{array}{l}\text { TRADITIONAL } \\
\text { AIR COOLED } \\
\text { DATA CENTER } \\
\end{array}$ & $\begin{array}{l}\text { PLATE LIQUID } \\
\text { COOLING } \\
\text { DATACENTER } \\
\end{array}$ & $\begin{array}{l}\text { FULLY IMMERSED } \\
\text { LIQUID-COOLED } \\
\text { DATACENTER } \\
\end{array}$ \\
\hline $\begin{array}{l}\text { Maintenance } \\
\text { Mode }\end{array}$ & Traditional Way & Traditional Way & Boom Lifting Maintenance \\
\hline Cryogen & $\begin{array}{l}\text { Gradually use R410A } \\
\text { environmentally } \\
\text { friendly refrigerant }\end{array}$ & $\begin{array}{l}25 \% \text { Dowtherm SR-1 } \\
\text { and } 75 \% \text { deionized } \\
\text { water mixture }\end{array}$ & Novec7000 Coolant \\
\hline Site Utilization & $\begin{array}{l}\text { Face to face or back } \\
\text { to back, with regular } \\
\text { spacing of } 1.2 \text { meters }\end{array}$ & $\begin{array}{l}\text { Semi-liquid cooling } \\
\text { adopts modularization } \\
\text { and can be enclosed } \\
\text { in hot and cold aisles. } \\
\text { Full liquid cooling } \\
\text { adopts closed cabinet, } \\
\text { can adopt } \\
\text { micro-module or } \\
\text { direct arrangement }\end{array}$ & $\begin{array}{l}\text { Face-to-face or back-to-back, } \\
\text { the distance between the } \\
\text { face-to-face cabinets should } \\
\text { allow mobile mechanical } \\
\text { cranes to pass, and the } \\
\text { distance between the } \\
\text { back-to-back cabinets should } \\
\text { allow inspectors to operate, } \\
\text { and the regular spacing is } 1.8 \\
\text { meters }\end{array}$ \\
\hline Ground Flatness & $\begin{array}{l} \pm 4 \mathrm{~mm} \text { error per } \\
\text { square meter }\end{array}$ & No request & $\begin{array}{l}\text { Should not exceed plus or } \\
\text { minus } 3 \mathrm{~mm} \text { per square meter }\end{array}$ \\
\hline Drainage & $\begin{array}{l}\text { Consistent with } \\
\text { specifications }\end{array}$ & $\begin{array}{l}\text { The cabinet has a drip } \\
\text { tray, which needs to } \\
\text { be connected to the } \\
\text { drainage pipe of the } \\
\text { machine room }\end{array}$ & $\begin{array}{l}\text { Liquid collection ditch and } \\
\text { drainage ditch should be set } \\
\text { separately }\end{array}$ \\
\hline Floor Height & $\begin{array}{l}\text { Not less than } 4.2 \mathrm{~m} \\
\text { floor height }\end{array}$ & No request & $\begin{array}{l}\text { Single-phase immersion } \\
\text { liquid cooling, the floor } \\
\text { should be designed for falling }\end{array}$ \\
\hline $\begin{array}{l}\text { Floor Load } \\
\text { Bearing }\end{array}$ & $\begin{array}{c}\text { IT area } \\
8-10 \mathrm{KN} \text { per square } \\
\text { meter } \\
\end{array}$ & $\begin{array}{c}\text { IT area } 9.8-11.8 \mathrm{KN} \\
\text { per square meter }\end{array}$ & $\begin{array}{l}\text { IT area not less than } 13 \mathrm{kN} \\
\text { per square meter }\end{array}$ \\
\hline $\begin{array}{l}\text { Cooling Water } \\
\text { Pipe Material }\end{array}$ & Seamless steel pipe & 304 stainless steel & 314 or 316 stainless steel \\
\hline PUE & High & Relevant Low & Low \\
\hline
\end{tabular}

It can be seen from the table that the full-immersion liquid-cooled data center has the best effect in reducing energy consumption, but its site utilization rate is low, and it has 
strict requirements on ground flatness, floor height, floor load-bearing, etc., has a higher cost, and is more universal Poor liquid cooling.

\section{Conclusion}

With the application of massive smart devices, all kinds of user data are exploding, bringing new challenges to the construction of big data centers. New types of data centers are developing in the direction of high density and high performance. This article compares and analyzes high-density data centers. Different from traditional data centers and existing problems, a high-density data center three-tier infrastructure is proposed. A SOA-based data center software and hardware integrated management model is proposed to implement a unified hierarchical management of high-density data center resources. In view of the small space, high server power, and high-reliability requirements of high-density data centers, technologies such as high-voltage direct current and liquid cooling have been studied to better match the infrastructure layer of high-density data centers with the environment, achieving high resource utilization and saving The purpose of space and energy saving provides new ideas for the deployment of high-density data centers.

\section{Acknowledgment}

This study is supported by project"Key Technology Research of Software and Hardware Synergy Optimization of High-density Data Center Infrastructure".

\section{Reference}

1. Guo Jiapeng.Infrastructure and key technologies of Power data center based on cloud computing[J]. Electronic Component and Information Technology. 2019,3(12):81-82+93.

2. Wang Dewen.Infrastructure and key technologies of Power data center based on cloud computing[J].Automation of Electric Power Systems. 2012(3):15-19.

3. Meng Fanli.The Design and Implementation of University's Hyper-Converged Infrastructure Data Center [J].Information Technology and Informatization. 2019, 000(005):172-176.

4. Zhang Xiaoliang, Xie Fei, Cui Shuo, etc. Power Cloud Computing Data Center Based on Virtualization and Distributed Technology[C]// Power Industry Informationization Annual Conference. 0.

5. Li Bang. Analysis of key energy-saving technologies for cloud computing data centers[J]. Electronic Testing, 2017, 000(016):61-62.

6. Wang Yajun, Zhang Huimin. Deepening Application of Virtualization Technology in Power Industry[J]. Popular Electricity, 2015, 276(S2):159-163.

7. Mcfarlane R . Cooling the Green Data Center: Practical strategies for efficiently powering and cooling modern high-density data centers[J]. EC\&M, 2012.

8. Wang Lixin, Chang Lin. Design of refrigeration and air conditioning system for high-density data center[J]. Refrigeration Technology, 2015, 000(001):48-53.

9. Liu Xiaoqian, Yang Shoubao, Guo Liangmin, et al. Snowflake structure: a new type of data center network structure[J]. Chinese Journal of Computers, 2011.

10. Lao Yimin. Analysis of changes in the environmental requirements of the data center host room[J]. Computer Room Technology and Management, 2015(1):30-32.

11. Ma Liang. Analysis and design of modern data center system structure[D].2019 
12. Guo Liang, Qian Shengpan, Research and Development Analysis of Data Center Architecture and Integration Optimization [J].Information and Communication Technology and Policy,2019(2):1-5

13. Li Qian. Technical Research on High Voltage DC and Traditional UPS Power Supply System of Data Center[J]. Electronics World, 2020, No.598(16):7-8.

14. Xie Lina, Guo Liang.Discussion on liquid cooling technology and its development[J].Information and Communication Technology and Policy,2019.

15. XIAO Xinwen. Energy saving analysis of direct contact cold plate liquid cooling in data center[J].Building Science, 2019, 035(006):82-90.

16. Wang Shaopeng,Wang Shuling.Research on Energy Efficiency Index and Energy Consumption Model of Data Center[J].Telecommunications Network Technology,2019, 000(002):18-21. 